\title{
Some observations on the relation between intraocular pressure reduction and the progression of glaucomatous visual loss
}

\author{
MICHAEL SCHULZER, 'FREDERICK S MIKELBERG, ${ }^{2}$ \\ AND STEPHEN M DRANCE ${ }^{3}$ \\ From the 'Departments of Statistics and Medicine, the University of British Columbia, the ${ }^{2}$ Department of \\ Ophthalmology, St Paul's Hospital, Vancouver, and the ${ }^{3}$ Department of Ophthalmology, University of British \\ Columbia, Vancouver, BC, Canada
}

SUMmARY A quantitative measure of visual field loss associated with kinetic perimetry in chronic open-angle glaucoma (COAG) is discussed. With this new technique an analysis of covariance is applied to a retrospective study of $41 \mathrm{COAG}$ patients. It suggests that reduction of intraocular pressure may not be uniformly effective in controlling the progression of the disease.

When the visual fields of a patient with chronic openangle glaucoma are determined by kinetic perimetry, they show considerable variation with time in the number of scotomas, their size, and density. It is therefore not an easy matter to assess the progression of the disease in a given eye over successive perimetric examinations. Thus the efficacy of treatment is difficult to establish, and only marked changes in the visual field can be noted.

It was our aim to try to develop, on the basis of manual perimetric measurements, a reasonable quantitative summary which might be representative, specific, and consistent in its assessment of the patient's visual field. This quantification method of the 'scotoma mass' has been fully described by us elsewhere. ${ }^{12}$

\section{Materials and methods}

A retrospective sample of 41 eyes of 41 patients with chronic open-angle glaucoma was obtained by reviewing computerised records which were readily available. ${ }^{12}$ To qualify for inclusion in this sample the eyes had to have paracentral or arcuate defects within the central $30^{\circ}$ as well as two or more years of followup after the development of visual field defects. There were 23 men and 18 women. Their median age at the start of the study was 61.75 years (first quartile

Correspondence to Dr M Schulzer, Department of Ophthalmology, University of British Columbia, 2550 Willow Strect, Vancouver, BC, Canada V5Z 3N9
$55 \cdot 75$, third quartile $68 \cdot 25)$, and median follow-up time was 96 months (first quartile $49 \cdot 5$, third quartile 120).

The visual fields were recorded with the Tübinger perimeter by both kinetic and static techniques. Only patients whose initial visual acuity was $6 / 9$ or better and those whose final visual acuity was $6 / 12$ or better were studied. By means of this cut-off the amount of extraneous change was minimised. The Zeiss video plan was used to measure by planimetry the areas of the scotomas within the central $30^{\circ}$. On each eye a large set of observations was available over time, consisting of multiple manual perimetric determinations by experienced technicians, numerous intraocular pressure (IOP) determinations interspersed throughout the follow-up period, visual acuity measurements, details of treatment, and other data.

The sequence of stimulus strengths used in our study to explore the scotomas was $10,16,32,64,100$, 320,640 , and 1000 apostilb, with a 10-minute size target on a 10-apostilb background. Since the brain tends to perceive intensity changes logarithmically, ${ }^{3}$ as well as for statistical validity, stimulus intensities were re-expressed in log-apostilb (='decibel') units.

The total mass of all excavations (scotomas) identified as glaucomatous was evaluated as described. ${ }^{2}$ This yielded a final quantity $\mathrm{M}$, in $\mathrm{mm}^{2} \times$ decibel units, which measured the total visual loss due to scotomas ('scotoma mass') at a given time. It increased with the number of scotomas, with their traditionally measured areas to specific stimulus 
intensities, and with their depths in terms of the logarithm of the stimulus intensities used to identify them. It was found to outperform these univariate measures (number of scotomas, traditional areas, intensities) in terms of its longitudinal correlation with time, continuous availability of information over time (individual scotomas can usually be followed for a limited time only and thus continuity is lost), and a continuous range of values (unlike, for example, the discrete count of the number of scotomas).

\section{Results}

The relationship between scotoma mass and the intraocular pressure was examined in the 41 patients. Between any two successive perimetric determinations of the visual field a number of intraocular pressure readings were available. The means and standard deviations of these sets of readings were obtained, as well as the maximum intraocular pressure observed during such a period. By means of analysis of covariance ${ }^{4}$ the regression of scotoma mass was determined longitudinally (within patients) on the mean intraocular pressure recorded over the period just prior to the given perimetric determination. A highly significant negative correlation was observed between these two quantities $(p=0.003)$. Low mean IOP values tended to correspond to high values of scotoma mass (Fig. 1).

A similar negative relationship was found between the scotoma mass and the maximum IOP reading in the preceding time interval. No correlation

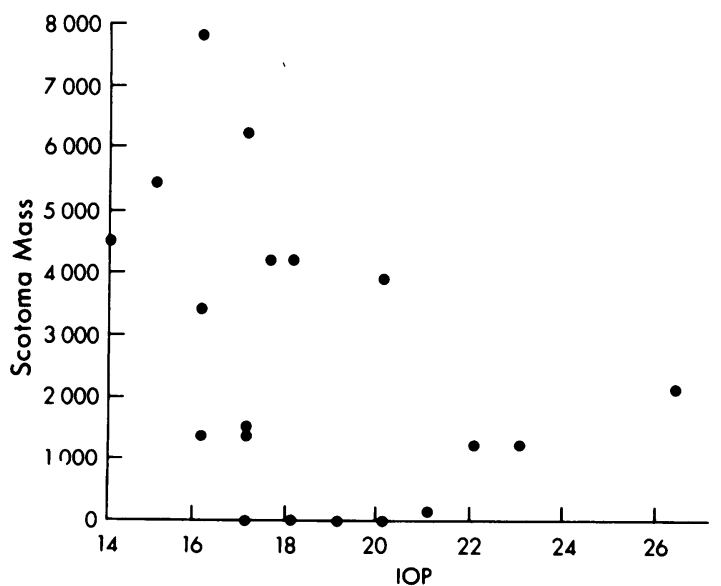

Fig. 1 Example of the correlation between intraocular pressure and total scotoma mass in one of the patients. The individual points are presented according to pressure level but independently of time. The scotoma mass and the intraocular pressure show a great variation, and there is a negative correlation between them. was observed between the scotoma mass and the logarithm of the standard deviation of the IOP readings $(p=0 \cdot 72)$.

The longitudinal regressions of mean IOP on time indicated significant negative slopes $(p=0.00005)$, with considerable interpatient variation.

The slope of scotoma mass over time for each patient was examined against the corresponding mean IOP time slope (Fig. 2). No significant correlation was found $(\mathrm{p}=0.42)$.

\section{Discussion}

Specific quantitation of glaucomatous visual loss has been difficult in ophthalmological practice until the recent advent of automatic perimetry..$^{56} \mathrm{We}$ developed a single, quantitative assessment of the total 'mass' of glaucomatous field loss. It represents, essentially, the sum of products of areas of scotomas in the inner $30^{\circ}$ of the field by their depths, where depths represent the logarithm of corresponding stimulus intensities used to explore the scotomas.

Our study of this scotoma mass measurement suggests that it possesses desirable statistical properties (symmetric distribution with light tails), which permit the use of standard analytical tools with reasonable validity.

The relationship between the scotoma mass and the IOP was explored. A strong, highly significant, consistent negative correlation was found between the mass of the scotomas and the IOP in the longitudinal analysis. For no patients could a positive correlation be found between the scotoma mass and

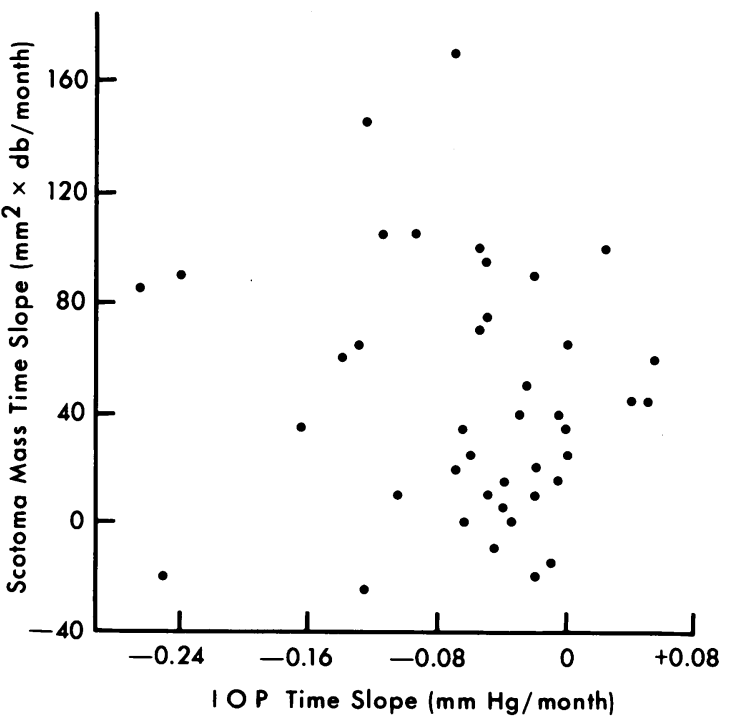

Fig. 2 Scatterplot of scotoma mass time slope for each patient against the corresponding IOP mean time-slope. 
the IOP. In the only other major study on progression Hart and Becker ${ }^{7}$ also found no correlation between IOP and the course of glaucomatous visual field defects.

Our data must be interpreted with caution. The sampling method excluded patients whose follow-up was too short (less than two years), and it was focused in the main on progressive cases. Extrapolation outside these limits should be avoided. Moreover, it is likely that in the management of these patients it was decided to treat the pressure more vigorously if it appeared to have been too high. One would also be inclined to respond more aggressively if the visual field loss had progressed. These factors may affect the relationship between the scotoma mass rate and IOP rate. However, the finding that the mass rates of change and the IOP rates of change were in fact independent may obviate this difficulty.

It is also possible that, as the disease advances and the scotoma mass increases, a natural burn-out can occur in some cases, which results in a pressure reduction with time. The pre-eminence of the role of IOP in glaucoma has recently been questioned by Krakau et al. ${ }^{8}$ Our data showed no positive pressuremass correlations. Only a carefully designed, pro- spective study could help untangle the correct interpretation of this finding. The recent computerisation of perimetry will make this task much easier.

This work was supported in part by MRC grant MT-1578 and the E A Baker Foundation for the Prevention of Blindness.

\section{References}

1 Mikelberg FS, Schulzer M, Drance SM, Lau W. Rate of progression of scotomas in glaucoma. Am J Ophthalmol 1986; 101: 1-6.

2 Schulzer M, Mikelberg FS, Drance SM. A study of the value of the central and peripheral isoptres in assessing visual field progression in the presence of paracentral scotoma measurements. $\mathrm{Br} J$ Ophthalmol in press.

3 Stevens SS. On the psychophysical law. Psychol Rev 1957; 64: 153-81.

4 Draper NR, Smith H. Applied regression analysis, 2nd ed. New York: Wiley, 1981.

5 Flammer J, Drance SM, Funkhauser A. Quantification of visual field defects with automated perimetry. Invest Ophthalmol Vis Sci in press.

6 Hirsch J. Statistical analysis in computerized perimetry. In: Whalen WR, Spaeth GL, eds. Computerized visual fields. Thorofare NJ: Slack, 1985: 309.

7 Hart WM Jr, Becker B. The onset and evolution of glaucomatous visual field defects, Ophthalmology 1982, 89: 268-79.

8 Krakau CET, Bengtsson B, Holmin C. The glaucoma theory updated, Acta Ophthalmol (Kbh) 1983; 61: 737-41.

Accepted for publication 13 August 1986. 